\title{
Les loisirs dirigés dans les collèges et les lycées
} (1937-1939)

Stimuler l'innovation pédagogique locale en attendant la réforme éducative

Directed leisure activities in secondary education - collèges and lycées

(1937-1939). Stimulating local pedagogical innovation while awaiting

educational reform

Die Freizeitgestaltung in den Sekundarschulen (collèges und lycées), 1937-1939.

Anspruch zur Erneuerung der Schule auf lokaler Ebene in Erwartung einer

Schulreform

Los tiempos de ocio dirigidos en los colegios e institutos (1937-1939). Estimular la innovación pedagógica local a la espera de la reforma educativa

\section{Jean-François Condette}

\section{OpenEdition}

Journals

Édition électronique

URL : https://journals.openedition.org/histoire-education/2303

DOI : 10.4000/histoire-education.2303

ISSN : 2102-5452

Éditeur

ENS Éditions

Édition imprimée

Date de publication : 1 janvier 2011

Pagination : $5-38$

ISSN : 0221-6280

Référence électronique

Jean-François Condette, «Les loisirs dirigés dans les collèges et les lycées (1937-1939) », Histoire de l'éducation [En ligne], 129 | 2011, mis en ligne le 01 janvier 2013, consulté le 20 mai 2021. URL : http:// journals.openedition.org/histoire-education/2303; DOI : https://doi.org/10.4000/histoire-education. 2303

(c) Tous droits réservés 


\title{
Les loisirs dirigés dans les collèges et les lycées (1937-1939)
}

\author{
Stimuler l'innovation pédagogique locale \\ en attendant la réforme éducative
}

\section{Jean-François CONDETTE}

" Nous avons été lancés dans une expérience hasardeuse, sans données précises, pour voir ce que cela donnerait. Or cette façon empirique de procéder n'est pas dans la manière française ${ }^{1}$ ", relève le proviseur du lycée de Lons-leSaunier dans un rapport qu'il transmet à l'inspecteur d'académie chargé d'opérer une synthèse sur la situation de l'enseignement secondaire en 1937-1938. Le proviseur fait ici référence aux loisirs dirigés lancés par Jean Zay, ministre de l'Éducation nationale du Front populaire. De son vaste plan de réforme de l'enseignement ${ }^{2}$, déposé comme projet de loi en mars 1937, peu de choses passent immédiatement dans la réalité de la classe, les exigences de l'heure fixant d'autres impératifs liés à la crise économique et à la situation internationale. Le jeune ministre a cependant le temps de proposer plusieurs innovations dont la classe d'orientation et les loisirs dirigés, mesures réformatrices qui sont de bons révélateurs de son idéal humaniste. Les loisirs dirigés tentent ainsi de créer, le samedi après-midi dans les collèges, les lycées et les écoles primaires supérieures ${ }^{3}$, un espace de liberté pédagogique qui doit permettre aux

\footnotetext{
1 Archives nationales, F/17/13932, rapport de l'inspecteur d'académie sur l'académie de Besançon, 2 décembre 1938, $35 \mathrm{p}$.

2 Archives nationales, 312 AP 5, Papiers Abraham, projets de réformes de l'enseignement, rapports dactylographiés.

3 Par nécessité de condenser le propos, nous focalisons l'analyse sur les collèges et les lycées.
} 
élèves et aux enseignants de travailler différemment, en s'inspirant des idées de l'Éducation nouvelle et des méthodes scoutes. Le constat fait par Jean Zay, rédigeant ses souvenirs dans sa cellule alors qu'il est emprisonné sur les ordres du régime de Vichy avant d'être assassiné par des miliciens en 1944, apparaît cependant trop optimiste lorsqu'il déclare :

"Dans le domaine proprement pédagogique, l'organisation des "loisirs ou activités dirigées" constitua la principale nouveauté. Le samedi après-midi fut réservé à une classe dans laquelle il serait fait appel de cent manières à l'activité spontanée de l'élève. Il s'agissait d'éveiller ses aptitudes ou ses dons, de favoriser ses goûts les plus sains, de le préparer à la vie, bref de rendre l'enseignement plus vivant [...]. L'imagination des maîtres, jointe à celle des élèves, aboutit, dans une atmosphère joyeuse et souvent enthousiaste, à des résultats étonnants [...]. Ce fut dans nos établissements une fenêtre largement ouverte, un appel d'air " ${ }^{4}$.

Créés par l'arrêté du 22 mai 1937, mis en place à partir de la rentrée d'octobre, les loisirs dirigés participent de cette " réforme scolaire expérimentale ${ }^{5}$ " qui doit assurer la promotion des idées nouvelles, tout en s'intégrant plus largement dans la politique de démocratisation de la culture et des loisirs que veut initier le Front populaire. Ils donnent lieu à une profusion d'initiatives mais rencontrent également de nombreuses oppositions, que n'évoque pas l'ancien ministre. Cet épisode pose dès lors la question, devenue récurrente, de l'articulation entre réforme politique et innovation pédagogique.

\section{I - La création des loisirs dirigés dans l'enseignement du Second degré et ses objectifs}

Dans l'enseignement du Second degré, le cabinet Jean Zay procède avec méthode mais sans avoir jamais les moyens financiers de ses volontés réformatrices ${ }^{6}$. Il s'agit à la fois de modifier les structures de cet enseignement et de rénover les pratiques pédagogiques.

4 Jean Zay, Souvenirs et solitude, Paris, 1946, réédition Belin, 2010 [Introduction d'Antoine Prost], p. 285.

5 Archives nationales, 312 AP 5, La Dépêche de Toulouse du 31 mars 1937.

6 Voir Pascal Ory, La Belle Illusion. Culture et politique sous le signe du Front populaire (1935-1938), Paris, Plon, 1994, chapitre XI : "Éducation », p. 613-711. 


\section{1 - Rénover les pratiques en créant un espace de libre initiative ?}

La réorganisation du ministère par le décret du $1^{\text {er }}$ mars 1937 est significative du sens de la réforme souhaitée. Le décret rattache les petites classes des lycées et des collèges à la direction du Premier degré et les écoles primaires supérieures à celle du Second degré. Les Écoles normales supérieures de SaintCloud et de Fontenay, qui couronnaient l'ordre primaire, sont confiées à l'Enseignement supérieur. Un système par degrés successifs doit ainsi remplacer l'ancien système fondé sur deux ordres juxtaposés, l'ordre primaire et ses prolongements (EPS et cours complémentaires ${ }^{7}$ ) et l'ordre secondaire et ses " petites classes ", tout en rapprochant les structures scolaires qui peuvent former l'enseignement "moyen " ${ }^{8}$. Le projet de loi déposé sur le bureau de la Chambre des députés le 5 mars 1937 va dans ce sens et s'inscrit dans l'esprit du projet d'École unique 9. "L'heure paraît venue de donner aux enseignements secondaire, primaire supérieur et technique le statut d'ensemble qu'ils attendent depuis de longues années " ${ }^{10}$. Il s'agit aussi de permettre la mise en place d'une réelle orientation à l'entrée dans le secondaire, alors que la gratuité, adoptée entre 1928 et 1933, fait craindre une forte hausse des effectifs et une baisse du niveau. Trois sections sont mises à la portée des élèves, classique, moderne et technique. Le travail d'Albert Châtelet, nommé directeur du Second degré par décret du 31 décembre 1936, est de mettre en œuvre cette réforme ${ }^{11}$. Le choix entre ces sections ne doit plus être le résultat du hasard ou de prédispositions sociales mais être lié à une décision réfléchie, fondée sur un bilan des compétences et des goûts de l'élève. C'est tout l'enjeu de la classe d'orientation qui est mise en place, à titre expérimental, par l'arrêté du 22 mai 1937 dans 45 centres en 1937-1938 et dans 35 centres l'année suivante ${ }^{12}$.

7 Jean-Pierre Briand et Jean-Michel Chapoulie, Les collèges du peuple : L'enseignement primaire supérieur et le développement de la scolarisation prolongée sous la Troisième République, Paris, CNRS-INRP, 1992, 544 p.

8 Jean-Michel Chapoulie, L'école d'État conquiert la France. Deux siècles de politique scolaire, Paris, Presses universitaires de Rennes, 2010, p. 351-370.

9 Jean-Michel Barreau, Jean-François Garcia et Louis Legrand, L'école unique (de 1914 à nos jours), Paris, Presses universitaires de France, 1998, 127 p.

10 Texte repris dans Henri Belliot, La réforme de l'enseignement; conférence faite par M. Jean Zay à l'Union Rationaliste, le 29 novembre 1937, Paris, Rieder, Les Éditions rationalistes, 1938, 123 p.

11 Jean-François Condette, Albert Châtelet : La République par l'École, Arras, Artois Presses Université, 2009, $602 \mathrm{p}$.

12 Voir Jean-Yves Seguy, Les politiques de démocratisation de l'enseignement secondaire dans l'entredeux-guerres ; des classes amalgamées aux classes d'orientation, réformes et réactions aux réformes dans le monde politique et éducatif français, thèse de doctorat (André D. Robert dir.), Université Lyon 2, 2010, $586 \mathrm{p}$. 
La volonté réformatrice de l'équipe Jean Zay touche également les pratiques pédagogiques. Il s'agit de rénover les méthodes d'enseignement, trop marquées par le cours ex cathedra et le bachotage de programmes démesurés. Le maître ne devra jamais perdre de vue qu'il lui appartient de favoriser "l'effort libre et spontané des élèves ${ }^{13}$. Les instructions du 30 septembre $1938^{14}$, qui s'appliquent aux lycées et collèges mais aussi aux écoles primaires supérieures et aux cours complémentaires, insistent sur cette dimension humaniste de l'enseignement secondaire, qui doit former un homme à même de comprendre le monde ${ }^{15}$.

"Son rôle est moins de les pourvoir d'un bagage de connaissances utiles que de favoriser le libre et complet développement de leurs facultés et d'en faire des hommes, en cultivant chez eux tout ce qui fait l'excellence de l'homme : l'intelligence, le cœur, le caractère, le sens moral, le goût du beau "16.

Il s'agit d'apprendre aux élèves à " savoir démêler le vrai du faux ", à raisonner sur les choses et à embrasser une question complexe. Ils doivent être capables d'exprimer par la parole et par la plume ce qu'ils ont à dire. La méthode d'autorité doit être étrangère à l'esprit de cet enseignement et les enseignants doivent habituer " les élèves à voir par leurs yeux et à penser de leur chef ". Il s'agit d'apprendre à apprendre. On doit les habituer à " manier les outils, à consulter les dictionnaires ", leur montrer " comment on prend des notes, comment on tient un cahier et un carnet de lectures ". C'est dans ce contexte que les réformateurs vont tenter d'insuffler dans les classes du secondaire, quelques-unes des idées et des pratiques revendiquées par les mouvements de l'Éducation nouvelle. Sans révolutionner le système, l'objectif est de fonder dans le temps scolaire un espace propice à l'adoption des méthodes actives.

13 Archives nationales, 312 AP 5, circulaire sur le dédoublement des classes au-dessus de 35 élèves, 9 octobre 1936.

14 Antoine Prost, "Les instructions de 1938 ", dans Antoine Prost (dir.), Jean Zay et la gauche du radicalisme, Paris, Presses de Sciences Po, 2003, p. 193-208.

15 Archives départementales du Pas-de-Calais, 81J120, "Enseignement du second degré. programmes,

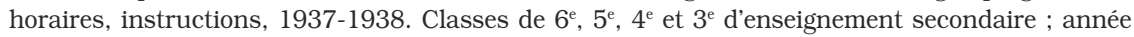
préparatoire, $1^{\text {re }}, 2^{\text {e }}$ et $3^{\text {e }}$ années d'enseignement primaire supérieur ", Fédération générale des pupilles de l'école publique, Paris, Bourrelier, 1938, 306 p.

16 Ibid., "préambule ", p. 5-13. 


\section{2 - La justification et l'organisation des loisirs dirigés : innover localement}

C'est l'arrêté du 22 mai 1937 qui institue les loisirs dirigés dans les collèges, les lycées et les EPS, en même temps que la demi-journée d'éducation physique de plein air.

"Article $1^{\mathrm{er}}$ : les horaires des classes des lycées et collèges, des EPS, sauf pour les classes de préparation aux grandes écoles, seront aménagés dans les conditions suivantes : $1^{\circ}$ Dans chaque classe, les heures d'enseignement d'une matinée ou d'un après-midi par semaine seront obligatoirement remplacées par une séance d'éducation physique en plein air. $2^{\circ}$ Aucun enseignement obligatoire ne sera donné le samedi après-midi.

Article 2 : L'après-midi du samedi est laissée à la disposition des établissements pour l'organisation de conférences, projections de vues, concerts, visites de musées, excursions, travaux manuels [...] et en général de toutes activités ayant pour objet de compléter, sous une forme récréative et selon le goût de chacun, la culture intellectuelle, esthétique et morale des élèves " ${ }^{17}$.

Le lien est évident avec la politique plus générale du Front populaire, qui assure la promotion des loisirs par les congés payés et le développement des auberges de jeunesse ${ }^{18}$ ou par le choix d'une politique culturelle volontariste en ce qui concerne, entre autres domaines, la recherche, le théâtre ou le cinéma. La circulaire du 8 juin 1937, écrite par Albert Châtelet, fixe ensuite les modalités pratiques d'organisation des loisirs dirigés dans le second degré.

" GÉNÉRALITÉS : "Les loisirs dirigés seront institués dans les premières semaines d'octobre 1937 dans les lycées et collèges. Je vous prie de rappeler à quelles nécessités pédagogiques ils répondent : 1 : Ils doivent offrir aux maîtres la possibilité de compléter la culture de leurs élèves par tels ou tels moyens exceptionnels qui ne s'accordent pas avec les conditions ordinaires de l'enseignement ; 2 : Ils doivent permettre, par l'organisation d'activités variées, de développer chez les élèves, les goûts et aptitudes individuelles qu'un enseignement à la fois collectif et spécialisé risque de négliger : travaux manuels, musique et dessin, recherches personnelles de toutes natures. De ce point de vue, la nouvelle institution peut-être utilisée pour le discernement des aptitudes et l'orientation. 3 : Enfin, en faisant appel à l'initiative des élèves, en provoquant leur groupement, pour des activités définies et concertées, ils doivent contribuer à leur éducation morale et à la formation en eux du sens social, à celle de la personnalité, du sens de la discipline et de la responsabilité collectives, du respect de l'intérêt général [...]

17 Archives départementales du Pas-de-Calais, $81 \mathrm{~J}$ 10, arrêté ministériel du 22 mai 1937 créant les loisirs dirigés.

Voir Pascal Ory, La belle illusion..., op. cit., chapitre XII, "Sports et loisirs ", p. 715-787. 
ORGANISATION : Les loisirs dirigés du samedi après-midi sont facultatifs pour les élèves. Ils peuvent être organisés de manières très diverses : excursions géographiques, géologiques, botaniques ; visites de musées, d'usines ou de fabriques ; conférences, projections lumineuses, cinématographiques, auditions musicales ; initiation aux travaux manuels, instruction musicale et artistique en général ; organisation de chorales, d'orchestres, organisation de fêtes, représentations théâtrales etc. ; activités de société ou cercles organisés et administrés par les élèves eux-mêmes : sociétés de spécialistes, naturalistes, historiques, collectionneurs, etc. ; lecture publique par les professeurs et les élèves ; salle de lecture ou bibliothèque librement ouvertes aux élèves... D'autre part, la radiophonie scolaire organisera le samedi après-midi, des concerts et des conférences particulièrement adaptées aux loisirs ${ }^{19}$.

Dans une circulaire du 20 octobre 1937, le ministère complète ses instructions. Ces loisirs seront organisés dans les classes de sixième, cinquième, quatrième et troisième, le cours préparatoire, les classes de première et de seconde année des EPS. Il est rappelé qu'ils sont "facultatifs pour les élèves " et que les classes primaires des collèges et des lycées ne sont pas comprises dans l'expérimentation. "Il est entendu qu'aucun changement n'est apporté le samedi après-midi au fonctionnement normal des services d'administration et de surveillance, études du soir comprises. Internes, demi-pensionnaires et externes surveillés ne peuvent s'absenter le samedi après-midi que sur la demande expresse des familles ". Il ne peut plus y avoir d'enseignement obligatoire le samedi après-midi mais il n'est pas question de fermer les établissements. Les élèves qui refusent les loisirs dirigés doivent continuer à être accueillis comme auparavant et le chef d'établissement doit prendre des mesures pour les occuper de 14 à 17 heures. "En principe, les élèves devront être libres de choisir l'activité qui leur convient le mieux ". Quand ils s'inscrivent comme membres d'un cercle, il est bon de ne pas exiger d'eux un engagement pour toute l'année. Il faut créer une commission des loisirs par établissement avec des représentants du personnel administratif, d'enseignement et de surveillance. Il n’y a que des " avantages à la représentation des associations de parents d'élèves et d'anciens élèves ainsi qu'à celle des élèves eux-mêmes ". La discipline doit aussi être adoucie.

" De même que les loisirs dirigés rompent avec certaines habitudes traditionnelles de l'enseignement, de même ils peuvent être l'occasion d'initiatives prudentes concernant le régime disciplinaire. La formation de cercles, sociétés, troupes d'excursionnistes permet l'apprentissage de la liberté individuelle. Les chefs d'établissement jugeront, si dans certains cas, la surveillance des salles ne peut pas être confiée aux élèves eux-mêmes. En tout cas, un effort doit

19 Archives départementales du Pas-de-Calais, 81 J 10, circulaire ministérielle du 8 juin 1937. 
être fait pour que les élèves se sentent, au moment des loisirs, sous un régime différent du régime traditionnel $»^{20}$.

L'arrêté du 6 août 1937 sur la réduction des horaires de l'enseignement dans les EPS précise les réductions rendues nécessaires par la demi-journée de plein air et l'après-midi de loisirs dirigés ${ }^{21}$. Un autre arrêté du même jour fait de même pour les horaires des classes des lycées et collèges selon les séries ${ }^{22}$. L'incitation ministérielle se fait ici impérative par l'imposition d'un cadre pédagogique novateur au sein de tous les établissements secondaires mais l'expérience se veut aussi respectueuse d'une certaine forme d'autonomie et de droit à l'initiative locale dans le détail des actions menées. Cette ambivalence entre imposition forcée d'une innovation et promotion du droit à l'initiative devait être souvent relevée par les opposants aux loisirs dirigés. La décision de rendre facultative la participation des élèves à ces activités innovantes devait aussi, à brève échéance, entraîner bien des problèmes dans l'existence des loisirs dirigés.

\section{3 - Le scoutisme et l'Éducation nouvelle comme sources d'idées et de pratiques}

Dans ces réformes, on devine l'influence des mouvements liés à l'Éducation nouvelle comme celle du scoutisme. Depuis la fin du XIX siècle au moins, divers pédagogues et mouvements pédagogiques réclament une réforme profonde des systèmes éducatifs européens pour faire davantage de place aux connaissances issues de la psychologie de l'enfant et de l'adolescent ou aux expérimentations pédagogiques. La promotion du jeu, de la libre initiative des élèves, l'ancrage des apprentissages dans le réel, la prise en compte des centres d'intérêts et des goûts des élèves sont les idées majeures de ces mouvements qui tentent difficilement, pendant l'entre-deux-guerres, de fédérer leurs initiatives tout en appliquant leurs idées au sein d'écoles spécifiques ${ }^{23}$. On peut aussi, évidemment, faire le lien avec certaines des idées de Célestin Freinet, qui tente lui aussi de promouvoir les méthodes actives,

20 Ibid., circulaire du ministre de l'Éducation nationale aux recteurs et inspecteurs d'académie, 20 octobre 1937.

21 Ibid., arrêté du 6 août 1937 portant réduction des horaires de l'enseignement dans les EPS.

$226^{\mathrm{e}} \mathrm{A}$ (21 heures), $6^{\mathrm{e}} \mathrm{B}$ (19 heures), 5e $\mathrm{A}$ (21 heures), $5^{\mathrm{e}} \mathrm{B}$ (19 heures), 4 $\mathrm{e}$ (22 heures), 4 $\mathrm{A}^{\prime}$ (21 heures), $4^{\mathrm{e}} \mathrm{B}$ (20 heures), $3^{\mathrm{e}} \mathrm{A}$ (22 heures 30 minutes), $3^{\mathrm{e}} \mathrm{A}^{\prime}$ (22 heures 30 minutes), $3^{\mathrm{e}} \mathrm{B}$ (22 heures).

23 Voir Annick Ohayon, Dominique Ottavi et Antoine Savoye (dir.), L'Éducation nouvelle, histoire, présence et devenir, Bern, Peter Lang, $2^{\text {de }}$ éd., 2007, 330 p. 
ou avec le mouvement de Roger Cousinet, qui fait la promotion du travail libre par groupes. L'influence du mouvement scout apparaît plus importante encore. Le nouveau directeur de l'Enseignement du second degré, Albert Châtelet, s'intéresse depuis longtemps au développement du mouvement scout laïque, y plaçant ses propres enfants. Il est choisi en 1937, l'année même du lancement des loisirs dirigés, comme président des Éclaireurs de France $(\mathrm{EDF})^{24}$ et prend ainsi la succession de Georges Bertier, le directeur de l'école des Roches. Dès lors, les liens entre le mouvement scout et l'Éducation nationale sont renforcés, le ministère favorisant le développement du mouvement par une habile promotion dans les établissements alors que le mouvement scout aide à la mise en œuvre des réformes scolaires. De multiples responsables des EDF viennent ainsi en aide pour former les cadres des auberges de jeunesse et des colonies de vacances ${ }^{25}$. Baden Powell, le fondateur du scoutisme, est reçu par le président de la République et Jean Zay en Sorbonne, en décembre 1936, pour célébrer le vingt-cinquième anniversaire des Éclaireurs de France. Jean Zay insiste alors sur la filiation entre ses réformes et l'esprit du scoutisme :

"C'est qu'en effet, loin de se combattre, le scoutisme et l'école poursuivent des tâches convergentes [...]. Vous avez mis en pratique des méthodes d'éducation dont l'efficacité n'est plus à démontrer et dont la simplicité n'est pas le moindre mérite [...]. Ce qui fait la valeur de la pédagogie scoutiste, c'est qu'au lieu de combattre ce qu'il y a de spontané chez les enfants, elle l'utilise et le développe. Éduquer en utilisant les aptitudes naturelles, c'est aussi ce que nous essayons de réaliser sur un autre plan et dans une autre sphère. Nous voulons, dans toute la mesure du possible, un enseignement qui fasse appel à l'initiative de l'élève, à son esprit d'observation, qui lui permette, sous une direction avisée, de découvrir par lui-même ce qu'il s'agit de lui apprendre ${ }^{26}$.

En décembre 1937, Le Chef, journal des éclaireurs de France, destiné aux cadres du mouvement, consacre un numéro spécial aux Loisirs scolaires ${ }^{27}$.

24 Pierre Kergomard et Pierre François, Les éclaireurs de France de 1911 à 1951, Paris, EEF, 1983, $380 \mathrm{p}$.

25 Voir Nicolas Palluau, "La formation des moniteurs de colonies de vacances (1936-1939) " dans AnneMarie Châtelet, Dominique Lerch et Jean-Noël Luc (dir.), L'école de plein air : une expérience pédagogique et architecturale dans l'Europe du XX'e siècle, Paris, Éditions Recherches, 2003, p. 289-295. Voir aussi Nicolas Palluau, Former des cadres pour la jeunesse. Chefs éclaireurs, professeurs, instituteurs et moniteurs de colonies de vacances (1911-1940), Thèse de doctorat (Pascal Ory, dir.), Université Paris 1, 2010).

26 Pierre Kergomard et Pierre François, Les éclaireurs de France..., op. cit., p. 193-194.

27 Le Chef, journal des éclaireurs de France, numéro spécial, "Les loisirs scolaires ", n 181, décembre $1937,88 \mathrm{p}$. 
Il s'agit de 88 pages de conseils et de suggestions pour les loisirs dirigés. " Depuis plus de vingt-cinq ans, nous utilisons et guidons les loisirs de milliers d'enfants et d'adolescents ${ }^{28}$ ". La brochure est destinée à faire la synthèse des loisirs possibles. "Au fond du placard les livres! Lire est beau, lire est bon. Mais on a eu du livre cinq jours déjà. Un peu de variété dans le régime ! Qu'on ne sente plus l'odeur du papier imprimé ! On lui reviendra ensuite avec plus de respect, plus d'amour ! ". Il s'agit de laisser respirer les élèves, de les laisser se promener et découvrir la richesse de la nature. La suite de la brochure est très précise et explique la manière de répartir les élèves en groupes stables. Pour être véritablement éducatives, les activités doivent être programmées sur toute l'année pour être "attrayantes, variées et progressives ${ }^{29}$ ". Tout doit reposer sur l'appel à l'initiative des enfants et Le Chef propose alors une multitude d'activités. L'éducation artistique (musique, danse, théâtre, dessin, peinture, etc.) offre de nombreuses ressources. La fabrication de jeux, la construction de jouets, la connaissance des oiseaux, des arbres, les exercices pratiques d'orientation et de lecture d'une carte, sont autant de moyens ludiques d'enrichir l'univers intellectuel et moral de l'élève. Il est aussi possible de lancer des recherches en bibliothèque, de les initier à l'examen du paysage ou à la visite d'une usine. Tout ceci demande évidemment du matériel et des ressources financières. Il ne faut pas hésiter, signale la brochure, à faire appel aux familles. "Dans un établissement d'enseignement secondaire, bien peu de parents refuseront de payer une cotisation annuelle de vingt ou trente francs, s'ils savent que cette somme est utilisée pour donner à leurs enfants un complément de formation intéressant ${ }^{30}$ ". Il faut organiser une coopérative scolaire (vente de crayons, petits pains, etc.). La constitution d'une telle coopérative exige que l'on fasse appel à l'initiative des élèves et qu'on leur donne des responsabilités.

La routine ne doit jamais s'installer et il faut prévoir "l'imprévu ". Le mauvais temps, l'exercice organisé pour quarante alors qu'ils ne sont que douze, ne doivent pas mettre par terre la demi-journée. Il faut donc prévoir des activités de secours. Les pages 31 à 57 proposent ensuite des études de cas. La troisième, par exemple, explique la visite d'une usine à gaz qui n'est pas qu'une simple illustration du cours de chimie. Elle doit permettre de s'inté-

28 Ibid., p. 2.

29 Ibid., p. 18.

30 Ibid., p. 27. 
resser à l'organisation du travail et à la condition ouvrière. Cette brochure sur les activités dirigées devait être rééditée en mai puis en octobre 1938, signe de son succès. Un numéro plus tardif du Chef daté de février 1939, consacre cent onze pages au " Plein air ", c'est-à-dire à l'organisation de la demi-journée de plein air ${ }^{31}$. Paul Barrier, inspecteur général, revient dans l’introduction sur les loisirs dirigés :

"Les activités dirigées, auxquelles le nom primitif de loisirs dirigés a porté tort, sont trop souvent apparues comme une façon d'enseigner à occuper agréablement le temps perdu. [...]. On n'a pas vu, qu'au contraire, cette institution vise essentiellement à développer chez l'enfant le goût de l'activité libre et volontaire et qu'ainsi elle vient compléter heureusement un système d'éducation resté jusqu'ici trop purement intellectuel $»^{32}$.

Le mouvement scout apparaît alors comme "l'éclaireur " de la réforme qui donne à l'Éducation nationale les conseils pratiques mais aussi souvent les cadres humains capables de mettre en place ces innovations. Lors de l'exposition sur les loisirs dirigés au lycée Carnot de Paris, en juin-juillet 1938, une place est faire au scoutisme. "À gauche, le scoutisme, avec deux scouts prêts à vous donner renseignements et brochures ; nous y trouvons des photographies de camps agrandis, des panoramas, des études de terrains, des herbiers, des cartes du ciel, toute la signalisation du scoutisme 33 ". Comme la presse scoute, les maisons d'édition se mettent sur les rangs pour guider cette réforme et en tirer profit. Hachette lance ainsi en 1938 une collection intitulée "Loisirs dirigés ", alors que Bourrelier consacre son premier volume de la série des Cahiers de pédagogie moderne à "L'enseignement de la géographie ", en explicitant le possible usage de ces trois heures de libre pédagogie. La Ligue de l'enseignement publie, dès le début de l'année 1937-1938, des feuillets à bas prix (un franc cinquante) qui permettent de couvrir un large éventail d'activités.

31 Le Chef, journal des éclaireurs de France, numéro spécial consacré au " Plein air ", février 1939, $\mathrm{n}^{\circ} 194,112 \mathrm{p}$.

32 Ibid., p. 1 et 2.

33 Pierre Nardin, "L’exposition des loisirs dirigés ", Revue universitaire, 1938, p. 207-215. 


\section{II - La mise en œuvre des loisirs dirigés : une fenêtre sur la vie}

À partir d'octobre 1937, les initiatives sont multiples au sein des établissements secondaires pour mettre en œuvre l'arrêté du 22 mai. Il faut à la fois convaincre les chefs d'établissement, les enseignants, mais aussi les familles et les élèves, de l'intérêt des loisirs dirigés.

\section{1 - Typologie des actions menées dans et hors des établissements}

La circulaire du 20 octobre 1937, après avoir longuement décrit les modalités d'organisation, précise de nouveau la large gamme des loisirs dirigés possibles $^{34}$. Les rapports des inspecteurs d'académie (IA) sur la situation de l'enseignement secondaire dans chaque académie en 1937-1938 puis en 1938-1939 contiennent de nombreuses informations sur la mise en place, le développement mais aussi les limites des loisirs dirigés. Nous ne pouvons ici les reprendre tous. Quelques constantes se dégagent cependant que l'on peut synthétiser ${ }^{35}$.

\section{Activités artistiques}

Les activités artistiques occupent une place importante sous des formes très diverses. Elles ont aussi le grand avantage de pouvoir, pour la plupart, se passer au sein de l'établissement, sans exiger de moyens financiers trop importants et d'être finalement assez proches de la forme scolaire classique. Apparaissent ainsi dans de nombreux établissements des chorales, de petits orchestres, des groupes d'élèves travaillant sur les musiciens et les instruments, sur les compositeurs. Les activités de théâtre sont également multiples, avec la création de troupes qui montent des spectacles. Des théâtres de guignols ou de marionnettes sont mis en place et l'on fait souvent appel au lycée ou au collège de jeunes filles pour fabriquer les poupées, les vêtements et les décors. Le lycée Janson de Sailly a, par exemple, réalisé un magnifique théâtre de marionnettes avec ses élèves de quatrième. On trouve aussi des travaux sur les émaux, des vitraux, des ateliers de linographie, de la peinture

\footnotetext{
34 Archives départementales du Pas-de-Calais, 81J 10, circulaire ministérielle du 20 octobre 1937.

35 Voir aussi L'Information pédagogique, janvier-février 1938 (8 articles), mars-avril 1938, maijuin 1938, janvier-février 1939 (numéro sur le théâtre scolaire avec P. Bonnet-Dupeyron, " Une année de loisirs théâtre au lycée d'Amiens ", etc.).
} 
sur velours, des ateliers de poterie, de moulages. Le dessin et plus largement la peinture sont souvent proposés aux élèves. On peut aussi réaliser des fresques ou des expositions. Les archives Marcel Abraham contiennent ainsi divers documents, dont des photographies, sur les réalisations de plusieurs groupes d'élèves. Le petit lycée de Saint-Rambert (Rhône) a fondé divers groupes de loisirs dirigés, dont un groupe " décorateurs " : ceux-ci ont peint au réfectoire une vaste fresque sur la thématique, les loisirs offrant des représentations diverses (théâtre avec un personnage de Molière, sport avec l'équipe de football du lycée, etc.). Autour de cette activité picturale sont venues se greffer des causeries, des visites dans les musées et des lectures ${ }^{36}$. Dans l'académie de Nancy, orchestres et chorales se sont développés au lycée de Nancy, dans les collèges de Pont-à-Mousson, Lunéville, Verdun, Neufchâteau, Bruyères, ainsi que dans de nombreux établissements féminins ${ }^{37}$.

\section{Travaux manuels}

Les travaux manuels ont été très fortement stimulés et la gamme des activités est très large, depuis de petits travaux rudimentaires qui apprennent à manier la scie, le rabot, l'appareil à souder, jusqu'à des compositions beaucoup plus complexes centrées sur des ateliers de reliure ou d'électricité. Le petit lycée de Saint-Rambert, par son équipe de " constructeurs ", a ainsi travaillé sur les transports avec la fabrication de modèles réduits. Certains avaient choisi l'aviation et sont allés visiter le port aérien de Bron ; d'autres avaient fait le choix de l'automobile et sont allés visiter les usines Berliet; d'autres encore ont choisi les navires après une visite du port fluvial. Dans de nombreuses villes, l'occasion est ainsi donnée de rapprocher les établissements classiques des écoles primaires supérieures ou des écoles pratiques pour la mise en place commune d'activités utilisant le matériel technique ou professionnel de ces dernières. Au lycée Faidherbe de Lille, les activités dirigées apparaissent bien à la rentrée d'octobre 1937. Des activités de théâtre, de photographie, de découverte du modélisme ou des excursions géographiques ont été proposées. Le lycée fait aussi appel à l'Institut industriel du Nord de la France, qui met ses ateliers à la disposition des élèves de troisième et quatrième (une

36 Archives nationales, 312 AP 6, papiers Abraham, Loisirs dirigés, série de photographies sur le réfectoire.

37 Archives nationales, F/17/13932, rapport de l'IA de la Meurthe-et-Moselle sur l'académie de Nancy, 5 décembre 1938. 
cinquantaine) pour travailler le bois et le fer ${ }^{38}$. À Commercy, à Remiremont, l'inspecteur note des ateliers du bois et du fer. Au lycée de garçons de Nancy, un atelier de reliure a été fondé ${ }^{39}$. À Angoulême, " les jeunes filles se sont livrées à des travaux de broderie, de coupe, de travaux sur cuir" ${ }^{40}$ ".

\section{La TSF et le cinématographe}

Si les activités liées à la projection de films documentaires loués par l'établissement ou réalisés par les élèves au cours de leurs sorties rencontrent un vif succès, l'usage de la radiophonie ne semble pas apporter de satisfactions bien nombreuses. Dans le Doubs, l'inspecteur relève des essais, mais qui n’ont pas eu de succès " à cause des crachements étourdissants qui accompagnent la réception ${ }^{41}$ ". Au collège masculin de Saint-Lô, " une salle spéciale de ciné-loisir a été aménagée par la municipalité. Au lycée masculin d'Alençon, un second réfectoire a été transformé en salle de loisirs et foyer du lycéen ${ }^{42}$ ". Au lycée de jeunes filles du Havre, un important effort a été fait en faveur de la radiophonie. Une installation centrale d'écoute a été installée dans le bureau de la surveillante générale et trois haut-parleurs dans les salles. Dans l'académie de Nancy, la radiophonie est critiquée. "On se plaint d'un manque de variété et d'intérêt des programmes du samedi après-midi $^{43}$ ". L'inspecteur d'académie de la Roche-sur-Yon, pour l'académie de Poitiers, relève à propos de la TSF et du cinéma qu'il s'agit " de précieux auxiliaires des jours de mauvais temps ", mais il insiste aussi sur les limites techniques. Quelques établissements ont engagé de fortes dépenses pour équiper des salles (Saint-Jean d'Angély, Saint-Maixent, La Roche-sur-Yon, etc.) mais les résultats " ne sont pas en rapport avec les dépenses effectuées ${ }^{44}$ ". La qualité de l'audition reste médiocre et le programme des émissions est peu adapté à la jeunesse. Même propos sous la plume de l'inspecteur de l'Ain pour l'académie de Lyon : "L'enseignement par la TSF a causé beaucoup

38 Marc Dupire, Le lycée Faidherbe de Lille de 1918 à 1960, Université Lille 3, mémoire de maitrise, 1989, p. 89-90.

39 Archives nationales, F/17/13932, rapport de l'IA de la Meurthe-et-Moselle sur l'académie de Nancy, 5 décembre 1938.

40 Ibid., rapport de l'IA de La Roche-sur-Yon sur l'académie de Poitiers, 23 novembre 1938.

41 Ibid., rapport de l'IA pour le conseil académique de Besançon, 2 décembre 1938.

42 Ibid., rapport de l'IA d'Evreux pour le conseil académique de Caen, année 1937-1938.

43 Ibid., rapport de l'IA de la Meurthe-et-Moselle sur l'académie de Nancy, 5 décembre 1938.

44 Ibid., rapport de l'IA de La Roche-sur-Yon sur l'académie de Poitiers, 23 novembre 1938. 
de désillusions en dépit des installations réalisées parfois à grands frais. L'audition est en général mauvaise et incertaine [...]. Beaucoup d'émissions sont sans valeur [...]. Le débit est trop rapide ${ }^{45}$ ".

\section{Les activités et sorties en lien avec une discipline scolaire}

De nombreux loisirs dirigés tournent autour d'une discipline scolaire qu'il s'agit d'aborder différemment, autour de sorties et de travaux plus personnels des élèves. Au lycée de Montbéliard, l'inspecteur d'académie note de nombreuses visites, en particulier au musée d'histoire naturelle ${ }^{46}$. Dans de nombreux collèges et lycées, sont fondés des clubs de langues, qui s'intéressent à la civilisation du pays étudié. Au lycée de jeunes filles de Besançon, est créé un cercle littéraire qui étudie des textes classiques et travaille Le Bourgeois gentilhomme et un club de langues est très dynamique. Au lycée de jeunes filles du Havre, l'inspecteur apprécie " les thés de la section anglo-saxonne avec ses saynètes anglaises, ses jeux et ses chants accompagnés de disques ". Il y a aussi "dégustation de pudding anglais ". Les activités de la section d'histoire ancienne sont également dynamiques, avec des projections de constructions de maisons romaines ${ }^{47}$. À Niort, les élèves filles ont fait fonctionner un club d'enseignement ménager dirigé par la présidente des Anciennes élèves et ce club a été fort fréquenté car les élèves avaient le plaisir de déguster au goûter de quatre heures, les plats qu'elles avaient fabriqués ${ }^{48}$. Le collège masculin de Dieppe, qui a ouvert une section d'agriculture et une chorale, a aussi organisé de nombreuses excursions, en particulier des visites archéologiques et des visites de monuments ${ }^{49}$. Dans l'académie de Strasbourg, l'inspecteur relève de nombreuses sorties mais " le samedi se prête mal à certaines visites où le travail est généralement arrêté $^{50}$ ". Pierre Nardin insiste, dans sa description de l'exposition des loisirs dirigés organisée au lycée Carnot de Paris en juin 1938, sur leur grand intérêt pour les sciences naturelles, l'histoire et la géographie. "L'activité du naturaliste est une activité de plein air et se déroule à travers les bois, les champs, les carrières, les mines ${ }^{51}$ ". Les films d'excursion, la constitution d'herbiers,

45 Ibid., rapport de l'IA de l'Ain sur l'académie de Lyon, 18 décembre 1939.

46 Ibid., rapport de l'IA pour le conseil académique de Besançon, 2 décembre 1938.

47 Ibid., rapport de l'IA d'Evreux pour le conseil académique de Caen, année 1937-1938.

48 Ibid., rapport de l'IA de La Roche-sur-Yon sur l'académie de Poitiers, 23 novembre 1938.

49 Ibid., rapport de l'IA d'Evreux pour le conseil académique de Caen, année 1937-1938.

50 Ibid., rapport de l'IA de Metz sur l'académie de Strasbourg, 3 janvier 1939.

51 Pierre Nardin, "L'exposition des loisirs dirigés ", Revue universitaire, 1938, p. 207-215. 
de petits aquariums, de planches de minéralogie se sont multipliés. La géographie et l'histoire profitent aussi des loisirs dirigés. Signalons, par exemple, une grande carte sur toile des environs de Beauvais et une carte de densité de la population de l'Oise (lycée de Beauvais) ${ }^{52}$ ". Pour l'histoire, c'est surtout l'histoire locale qui a été valorisée : confection de maquettes de monuments, de plans, regroupement de photographies.

\section{2 - Les loisirs dirigés à connotation sociale}

Ces loisirs dirigés doivent donner aux élèves l'esprit de solidarité et sont fondamentaux dans la formation d'un citoyen actif et responsable. C'est souvent par la fondation de clubs, de cercles, d'associations que cet apprentissage de la citoyenneté solidaire se met en place. Le travail sur les statuts de l'association et sur la représentativité des élèves au sein des structures décisionnelles peut être poussé assez loin. La Société des naturalistes du lycée Carnot dispose ainsi de statuts précis. "Il est créé au lycée Carnot de Paris, sous la dénomination de Société des naturalistes du lycée Carnot, un groupement d'élèves s'intéressant aux sciences naturelles ${ }^{53}$ ". La société comprend des membres actifs (élèves des classes engagées dans les loisirs dirigés), des membres honoraires (élèves des classes supérieures) et des membres bienfaiteurs (anciens élèves, parents, etc.). Les buts de la société sont de " développer chez ses membres le goût et la pratique de l'étude de la nature et de permettre la récolte et la mise en collection de documents relatifs aux sciences naturelles et de façon générale, de favoriser toute manifestation intéressant ces sciences ". L'article IV insiste sur la gestion de l'association :

" [L]a direction de la société est assurée par les professeurs de sciences naturelles. Ils sont assistés dans cette tâche par un groupe de moniteurs chargés des détails et de la transmission des avis et des consignes [...]. Ils sont choisis parmi les élèves les plus capables, par leur savoir et leur caractère, d'aider et de guider leurs camarades les moins expérimentés ".

La cotisation est de dix francs pour un membre actif et de vingt francs au minimum pour un membre bienfaiteur. "Les sociétaires prennent l'engagement de se ranger avec docilité aux avis des moniteurs qui, de leur côté, doivent user avec tact et modération de l'autorité qui leur est momentané-

52 Ibid., p. 210.

53 Archives nationales, 312 AP 6, Papiers Abraham, Société naturaliste du lycée Carnot de Paris, Statuts. 
ment déléguée ". De nombreux rapports des chefs d'établissement, synthétisés par les inspecteurs d'académie, témoignent de la fondation de ce type d'association. Au lycée de jeunes filles de Lyon, huit groupes de loisirs dirigés ont fonctionné en 1937-1938. Un comité directeur a été fondé pour chaque groupe, en lien avec la commission centrale des loisirs qui se réunit pour établir le calendrier. "Les élèves présidents ont place en cette commission ainsi que des cheftaines des Éclaireurs et des anciens élèves ${ }^{54}$ ". L'inspecteur d'académie de la Haute-Marne, pour l'académie de Dijon, indique dans son rapport du 21 novembre 1938 : "Voici que peu à peu les loisirs s'organisent ; des cercles, des clubs, des ateliers groupent les élèves ${ }^{55}$ ". Dans l'académie de Strasbourg, les pratiques sont diversifiées. "Les élèves ont joué un rôle actif dans l'organisation des loisirs [...]. Ils ont apporté du matériel, dirigé les jeux, choisi le terrain [...]. Grâce à leurs initiatives personnelles, des questions de détail ont pu être réglées de façon efficace. Parfois, la direction des travaux a été confiée à des élèves moniteurs et avec succès [...]. Là, on n'a pas cru devoir se risquer à leur donner une part importante dans la direction même des séances, des expériences précédentes ayant enseigné qu'il faut être prudent et que l'apprentissage de l'initiative et de la responsabilité ne peuvent se faire que lentement [...]. Enfin, certaines commissions ont jugé bon de ne laisser aux élèves aucune part à l'organisation et à la direction des séances ${ }^{56}$ ".

Le journalisme scolaire, qui ne date pas de 1936, est largement valorisé par l'expérience ${ }^{57}$.

"Les feuilles abondent : La gazette, Le Bahut, Entre copains, Le courrier de la cinquième etc. Tous seraient à citer à cause de l'ardeur et de l'enthousiasme de leurs rédacteurs. Deux cependant me paraissent mériter une mention spéciale : Le Potache des Vosges (mensuel, imprimé au collège de Remiremont) et Le Messager (Lycée Henri-IV) " ${ }^{58}$.

De nombreux rapports témoignent de la fondation de tels journaux scolaires. Au lycée de Lons-le-Saunier, à côté d'un club philatélie et d'un groupe

54 Archives nationales, F/17/13932, rapport de l'IA de Lyon sur l'académie de Lyon, 8 décembre 1938.

55 Ibid., rapport de l'IA de la Haute-Marne pour l'académie de Dijon, 21 novembre 1938.

56 Ibid., rapport de l'IA de Metz pour l'académie de Strasbourg, 3 janvier 1939.

57 Voir Laurence Corroy, La presse des lycéens et des étudiants au XIXe siècle, Lyon, INRP, 2004, 280 p. ; Pierre Caspard (dir.), La presse d'éducation et d'enseignement, XVIII siècle-1940. Répertoire analytique, Paris, INRP-CNRS, 4 tomes : A-C, 1981, 560 p. ; D-J, 1984, 688 p. ; K-R, 1986, 566 p. ; S-Z et suppléments, 1991, $762 \mathrm{p}$.

58 Pierre Nardin, "L’exposition des loisirs dirigés ", Revue universitaire, 1938, p. 212. 
de construction de modèles réduits, a fonctionné un groupe chargé de la rédaction du journal des élèves, $L^{\prime} E ́ c h o ~ d u ~ b a h u t^{59}$. Au lycée de Cherbourg, on trouve un journal du lycée tirant à 350 exemplaires ${ }^{60}$.

L'organisation de coopératives " figure au premier plan des activités sociales. Le tableau des statuts de la coopérative du lycée de jeunes filles de Nîmes en explique à tous le fonctionnement et la réalisation : le bilan est impressionnant ${ }^{61}$ ". Il s'agit d'aider à l'envoi en colonies de vacances d'élèves dont la situation de famille est "digne d'intérêt ". Des sorties ont aussi été organisées vers les pupilles de l'Assistance publique et les malades des hôpitaux. Dans bien des établissements, on confectionne des habits pour les enfants nécessiteux. À La Rochelle, on organise un cercle hispanique au service des réfugiés basques. Au collège masculin de Dôle, on assiste à la fondation d'une " coopérative gérée par les élèves sous le contrôle du principal et dont les bénéfices sont utilisés pour les loisirs dirigés ${ }^{62}$ ". Le bonus pour 1937-1938 a été de 1000 francs. Au collège féminin de Lunéville, des élèves se sont lancées dans l'apprentissage de l'écriture braille au profit de l'œuvre des aveugles ${ }^{63}$. Dans l'académie de Strasbourg, de nombreux établissements de jeunes filles ont proposé des activités tournées vers la confection de vêtements et de jouets pour les enfants de la ville, cadeaux distribués ensuite aux élèves de certains quartiers défavorisés ${ }^{64}$.

\section{3 - Présence facultative des élèves et absentéisme}

Dès l'arrêté du 22 mai 1937, il est indiqué que les loisirs dirigés ne sont pas obligatoires mais facultatifs et qu'il faut maintenir des activités classiques, cours exclus bien évidemment, pour les élèves qui s'en abstiendront : travail en étude et en bibliothèque, etc. Dès lors, l'un des principaux défis des chefs d'établissements et des enseignants est de convaincre les élèves de l'intérêt des activités proposées. Pour cela, il est nécessaire à la fois de prévoir celles-ci assez longtemps à l'avance pour éviter l'improvisation et de rendre visible l'offre

59 Archives nationales, F/17/13932, rapport de l'IA pour le conseil académique de Besançon, 2 décembre 1938.

60 Ibid., rapport de l'IA d'Evreux pour le conseil académique de Caen, année 1937-1938.

61 Pierre Nardin, "L'exposition des loisirs dirigés ", Revue universitaire, 1938, p. 212.

62 Archives nationales, F/17/3932, rapport de l'IA, conseil académique de Besançon, 2 décembre 1938.

63 Ibid., rapport de l'IA de la Meurthe-et-Moselle sur l'académie de Nancy, 5 décembre 1938.

64 Ibid., rapport de l'IA de Metz sur l'académie de Strasbourg, 3 janvier 1939. 
proposée afin de permettre le choix des élèves. Dans certains établissements, on demande aux élèves de s'inscrire dans une activité pour toute l'année ou au moins le trimestre. Dans d'autres, tel le collège de Dôle, il n'y a pas de groupes rigides d'élèves mais une inscription en début de chaque semaine en fonction du programme ${ }^{65}$. Dans l'académie de Strasbourg, "souvent les élèves ont été priés de faire des vœux concernant les activités présentes dans le programme. Il en a été tenu compte dans la mesure du possible ${ }^{66}$ ".

Au collège de Dreux, la commission des loisirs, instituée en octobre 1937, constate rapidement que "le nombre des demandes d'inscription formulées par les élèves dépasse de loin toutes les prévisions ${ }^{67}$ ". Il n'en demeure pas moins qu'une fois l'attrait de la nouveauté passé, les effectifs semblent souvent décliner. Le rapport sur l'enseignement secondaire dans l'académie de Besançon, préparé pour le conseil académique du 2 décembre 1938, insiste certes sur la mise en place des loisirs dirigés dans de nombreux établissements mais note cependant que "le nombre des participants, très élevé au début, a diminué dans la suite ${ }^{68}$ ". Au lycée de Lons-le-Saunier, il relève 60 participants sur 314 élèves. L'inspecteur d'académie de l'Ardèche, chargé du rapport sur l'académie de Grenoble en 1937-1938, note que de nombreux maitres sont sur la réserve, mais il signale aussi que de nombreuses familles " préfèrent voir leurs filles consacrer leur samedi après-midi à des essayages ou à des séances chez le coiffeur. D'autres les confient à des patronnes privées ${ }^{69}$ ". Il relève également pour le lycée Champollion de Grenoble, de la sixième à la troisième, 140 inscrits en 1937-1938 sur 580 élèves. Dans l'académie de Bordeaux, le rapport de 1937-1938 signale : " pour si ingénieux que soient partout les efforts, la réforme a bien du mal à entrer dans les mœurs [...]. Les familles n'ont pas manqué d'opérer un rapport entre la faiblesse de certains résultats aux examens et l'extension donnée aux loisirs dirigés et à l'éducation physique ${ }^{70}$ ". L'inspecteur dément ces propos en signalant qu'aucune heure n'a été éliminée

65 Pierre Nardin, "L’exposition des loisirs dirigés ", Revue universitaire, 1938, p. 212.

66 Archives nationales, F/17/3932, rapport de l'IA de Metz sur l'académie de Strasbourg, 3 janvier 1939.

67 Etienne Colomb, "Impressions d'élèves sur les loisirs dirigés ", Revue universitaire, 1938, p. 36-39.

68 Archives nationales, F/17/13932, rapport de l'IA pour le conseil académique de Besançon, 2 décembre 1938.

69 Ibid., rapport de l'IA de l'Ardèche sur l'académie de Grenoble en 1937-1938, $1^{\text {er }}$ décembre 1938.

70 Ibid., rapport de l'IA du Lot-et-Garonne sur l'académie de Grenoble en 1937-1938, 8 décembre 1938. 
mais que l'on a reporté sur d'autres jours les enseignements autrefois placés le samedi après-midi, ce qui a surchargé ces journées. L'inspecteur de la HauteLoire, dans son rapport sur l'académie de Clermont, le 4 mars 1939, écrit que "la réforme a été généralement bien accueillie par les chefs d'établissement ${ }^{71}$ " mais que les élèves sont plus méfiants. "Les élèves, ça et là, ont pu envisager avec quelques hésitations le cadeau qu'on leur offrait ". Les internes ont dit " oui " en masse selon lui, à plus de $90 \%$, car ces loisirs sont vraiment un moyen d'aérer la vie scolaire. Du côté des externes, l'accueil a été beaucoup moins enthousiaste, avec des effectifs de volontaires oscillant entre 10 et $40 \%$. L'inspecteur de la Meurthe-et-Moselle, pour l'académie de Nancy, note que les chefs d'établissement sont " à peu près unanimes à reconnaitre l'intérêt et la valeur éducative de ces séances ${ }^{72}$ ". Leur caractère non obligatoire lui apparaît cependant dommageable. "On éviterait ainsi bien des absences et, au troisième trimestre, une désertion surtout massive chez les élèves externes dont la plupart veulent profiter du week-end ".

L'inspecteur qui écrit le rapport sur l'académie de Strasbourg signale, lui aussi, une participation décroissante. Au lycée masculin de Colmar, si deux tiers des élèves sont volontaires pour participer aux loisirs dirigés au premier trimestre, ils ne sont plus qu'un tiers au dernier, répartis de la manière suivante :

\begin{tabular}{|l|c|c|c|}
\hline & $\begin{array}{l}\text { Inscrits } \\
\text { dans les classes }\end{array}$ & $\begin{array}{l}\text { Inscrits aux loisirs } \\
\text { dirigés en novembre- } \\
\text { décembre 1937 }\end{array}$ & $\begin{array}{l}\text { Inscrits aux loisirs } \\
\text { dirigés au 4 juin 1938 }\end{array}$ \\
\hline Seconde & 54 & 24 & 08 \\
\hline Troisième & 52 & 33 & 13 \\
\hline Quatrième & 68 & 45 & 40 \\
\hline Cinquième & 74 & 52 & 22 \\
\hline Sixième & 56 & 42 & 101 \\
\hline Total & 304 & 196 & \\
\hline
\end{tabular}

Au lycée de jeunes filles de Strasbourg, la proportion de volontaires varie de 60 à $80 \%$ en sixième, de 30 à $50 \%$ en cinquième, de 10 à $15 \%$ en quatrième et se situe à moins de $10 \%$ dans les niveaux supérieurs ${ }^{73}$. L'inspecteur

71 Ibid., rapport de l'IA de la Haute-Loire sur l'académie de Clermont, 4 mars 1939.

72 Ibid., rapport de l'IA de la Meurthe-et-Moselle sur l'académie de Nancy, 5 décembre 1938.

73 Ibid., rapport de l'IA de Metz sur l'académie de Strasbourg, 3 janvier 1939. 
remarque également que la libération de cette demi-journée, avec un horaire qui restait sensiblement le même, a conduit à alourdir davantage les autres jours. Dans l'académie de Lyon, des absences croissantes sont aussi relevées. Au lycée de Saint-Étienne, la commission des loisirs a mis en place sept groupes (théâtre, construction de modèles réduits d'avions, excursionnistes, conférences musicales et chorales, travail manuel, collectionneurs, poésie), mais " après une période d'engouement qui s'est placée naturellement à l'origine, a succédé une période de nombreux abandons ${ }^{74}$ ". Au lycée Ampère de Lyon, les loisirs dirigés ont commencé le 6 novembre 1937 pour 200 volontaires environ, ce qui donne un cinquième de l'effectif total. L'atelier lié à la radiophonie a totalement échoué, de même que l'atelier de construction mécanique. L'activité théâtre, en revanche, a intéressé les élèves. Au lycée du Parc de la même ville, annexe exceptée, le nombre maximum de volontaires a été de 140 élèves. "À partir de Pâques, les vides ont été de plus en plus nombreux ", les heures du samedi après-midi étant utilisées par les parents pour les visites et excursions en famille. "L'année scolaire s'est terminée avec un effectif d'une cinquantaine d'élèves ". Dans l'académie de Rennes, le rapport du 8 décembre 1938 note " qu'un peu partout, les effectifs fondent progressivement jusqu'à s'anéantir en fin d'année ${ }^{75}$ ".

Au collège de Dreux, Étienne Colomb rend compte d'une initiative qui témoigne du souci de recueillir l'avis des élèves. En décembre 1937, on a donné deux sujets de composition française. En classe de troisième le sujet est le suivant: "Depuis le début de l'année scolaire sont organisées au collège des séances de loisirs dirigés. Les élèves qui participent à ces séances feront connaître si elles les intéressent et pourquoi ; ceux qui n'y participent pas justifieront sincèrement leur abstention ". En classe de quatrième, le sujet est un peu différent : "Un de vos amis, élève d'un autre établissement, vous a demandé si vous participiez, au collège, aux séances de loisirs dirigés. Dans votre réponse, vous lui ferez part de vos impressions sur cette innovation introduite dans votre vie scolaire. Si vous n'êtes pas inscrit parmi les élèves participant aux loisirs, faites savoir à votre ami pourquoi vous n’y participez pas ». Sur douze élèves de troisième, onze sont inscrits aux loisirs, alors qu'ils sont vingt sur vingt-deux en quatrième. Les abstentions sont diversement motivées. Un élève qui habite loin du collège fait chaque jour un long trajet à bicyclette et estime que l'ob-

74 Ibid., rapport de l'IA de Lyon sur l'académie de Lyon, 8 décembre 1938.

75 Ibid., rapport de l'IA de Rennes sur l'académie de Rennes, 8 décembre 1938. 
servation de la nature lui tient lieu de loisirs dirigés. Un autre est très franc : " le mot loisir signifie détente, et l'on ne se repose réellement qu'en faisant ce que l'on désire. Je préfère, moi, rester en étude, où je puis dessiner, lire, écrire et, au besoin, rêver ${ }^{76}$ ".

\section{III - D'importantes difficultés dans l'application d'une réforme contestée}

L'écho rencontré par les loisirs dirigés est dans l'ensemble assez favorable au sein du monde éducatif. Le lycée Carnot de Paris organise du 25 juin au 3 juillet 1938, pour "l'An I des Loisirs dirigés ${ }^{77}$ ", une grande exposition qui insiste sur la réussite de l'expérience. Dans une circulaire aux recteurs du $1^{\mathrm{er}}$ avril 1939, Jean Zay, par la plume d'Albert Châtelet, dresse un premier bilan assurément trop positif :

"Les loisirs dirigés dans le second degré, raillés parfois à leur apparition, recueillent un assentiment à peu près général après une année d'expérience. [...]. Le mot "loisirs" a été d'autant plus critiqué qu'il s'introduisait dans la terminologie scolaire à un moment où le travail est plus que jamais nécessaire. Il semblait indiquer malgré le sens exact que lui donnaient les circulaires, la volonté d'organiser l'amusement. L'expression "activités dirigées" a été mieux accueillie, mieux comprise [...]. Les familles, les professeurs, les élèves l'ont compris, les préventions sont tombées. La démonstration est faite que les loisirs complètent de la plus heureuse façon l'enseignement. Les élèves goûtent leur liberté, se plaisent à constituer des équipes, à lire, à chercher, à observer l'œuvre d'art, la nature, la vie, à prendre des initiatives " ${ }^{78}$.

Mais cela ne saurait cacher de multiples limites qui finissent par faire s'étioler cette initiative innovante, alors que, dans une partie de l'opinion, les loisirs dirigés sont amalgamés à un refus plus général de la politique du Front populaire.

\section{1 - Les problèmes financiers et matériels : le coût négligé de l'innovation}

La mise en place des loisirs dirigés est confrontée à des problèmes matériels, des moyens financiers conséquents n'étant pas dégagés pour cette innovation

76 Etienne Colomb, "Impressions d'élèves sur les loisirs dirigés ", Revue universitaire, 1938, p. 36-39.

77 Pierre Nardin, "L'exposition des loisirs dirigés ", Revue universitaire, 1938, p. 207-215.

78 Archives nationales, 312 AP 6, Papiers Abraham, circulaire aux recteurs du $1^{\mathrm{er}}$ avril 1939. 
pédagogique. Albert Châtelet le reconnaît d'ailleurs dans sa circulaire aux recteurs du $1^{\text {er }}$ avril 1939 : " un problème délicat reste à résoudre, celui de la rémunération du personnel qui sera réglé prochainement par un texte actuellement en préparation ${ }^{79}$ ". La plupart des rapports des chefs d'établissement et des inspecteurs insistent sur le fait que tout repose sur le bénévolat et que celui-ci finit par s'émousser. Le rapport sur l'académie de Besançon, daté du 2 décembre 1938, met en avant l'engagement des professeurs bénévoles et indique dans sa conclusion :

" Ainsi est-il permis de penser que, si un jour l'Éducation nationale ne demande pas à ses dévoués collaborateurs de mettre en application une expérience onéreuse, sans lui donner le moindre argent, les résultats seront excellents, puisque avec des moyens minimes et de la bonne volonté, les résultats furent certains dans quelques établissements. Mais attention : le dévouement n'est plus monnaie courante en l'an de grâce 1938 et quelques professeurs me disaient, qu'eu égard à leur investissement en temps et en énergie cette année, ils se refuseraient à être plus longtemps les dindons de la farce "80.

Les établissements tentent de trouver des solutions temporaires par la mise en place d'une petite cotisation demandée à chaque élève qui participe aux loisirs dirigés. On organise aussi des fêtes, des spectacles, des kermesses pour récolter des fonds. Au lycée de jeunes filles de Lons-le-Saunier, le club théâtre a organisé une grande fête dont la recette a été versée à la caisse des loisirs. On a aussi fait appel à la générosité des associations de parents d'élèves. Au collège de Dreux, Étienne Colomb note, lui aussi, l'obstacle du manque de crédits. "On eut recours à une légère cotisation, à laquelle les élèves consentirent sans difficulté. D’autre part, des séances de cinématographe à prix réduit furent prévues pour obtenir quelques ressources supplémentaires $^{81}$ ". L'inspecteur de la Haute-Loire, note à la fin de son rapport sur l'académie de Clermont, le "redoutable écueil financier ${ }^{82}$ ". Les caisses des loisirs, les coopératives ne peuvent suffire si l'on ne veut pas se contenter de "bouts de ficelles ". L'inspecteur de la Meurthe-et-Moselle dit la même chose pour l'académie de Nancy : "les loisirs dirigés qui doivent être variés et intéressants [...] ne peuvent être bien organisés sans ressources impor-

79 Archives nationales, 312 AP 6, Papiers Abraham, circulaire aux recteurs du $1^{\mathrm{er}}$ avril 1939.

80 Archives nationales, F/17/13932, rapport de l'IA de Besançon, 2 décembre 1938, 35 p.

81 Etienne Colomb, "Impressions d'élèves sur les loisirs dirigés ", Revue universitaire, 1938, p. 36.

82 Archives nationales, F/17/13932, rapport de l'IA de la Haute-Loire sur l'académie de Clermont, 4 mars 1939. 
tantes ${ }^{83}$ ". Dans l'académie de Strasbourg, " souvent il est fait appel à des personnalités étrangères : conférenciers, président du syndicat d'initiative, présidents des clubs et associations de la ville ${ }^{84}$ ". Tout ceci a un coût, alors que l'on manque aussi de locaux une fois les salles de dessin et de gymnastique réquisitionnées. Les activités manuelles exigent des outils et des ateliers qui, bien souvent, sont manquants s'il n'y a pas à proximité une école pratique de commerce et d'industrie ou un établissement technique. Dès lors, même les partisans convaincus des loisirs dirigés finissent par se désengager, alors que la gauche pédagogique, mais aussi politique, dénonce de plus en plus le fait qu'une bonne idée est sacrifiée par le manque de moyens.

\section{2 - Acteurs éducatifs, enjeux corporatistes et méfiance face à l'innovation}

Un certain nombre d'administrateurs et d'enseignants avouent ne pas trop comprendre les modalités de mise en place des loisirs dirigés, qui sont à la fois obligatoires pour les établissements mais optionnels pour les élèves. La méthode gouvernementale gêne certains de ces acteurs en ce qu'elle adopte une pratique souple et expérimentale qui sort de l'ordinaire, comme le regrette le proviseur de Lons-le-Saunier ${ }^{85}$. Les enseignants eux-mêmes sont confrontés à diverses difficultés. Le rapport de l'inspecteur d'Évreux sur l'académie de Caen en 1937-1938 insiste sur les limites du bénévolat et sur l'instabilité des équipes qui détruit les dynamiques lancées. Telle section de sciences naturelles du lycée de garçons d'Évreux, qui a obtenu un grand succès l'an dernier, ne fonctionne plus aujourd'hui du fait du départ de l'enseignant.

"L'organisation des loisirs ou activités dirigés dans l'enseignement du second degré s'est opérée le samedi après-midi dans tous les établissements de l'académie de Caen. Le personnel, surpris par la nouveauté de cette forme d'éducation, s'est mis résolument à la tâche. Nombreux sont les petits collèges à personnel restreint où les loisirs ont été organisés à titre bénévole sans rétribution aucune [...]. Mais on s'est vite aperçu, si l'on ne voulait pas toujours parcourir les mêmes sentiers battus, que l'organisation des loisirs dirigés exigeait, de la part des professeurs, chargés d'un groupe d'activités [...] un effort de programmation et de mise au point assez précise. De là des plaintes nombreuses sur le surcroît de travail imposé au personnel $" 86$.

83 Ibid., rapport de l'IA de la Meurthe-et-Moselle sur l'académie de Nancy, 5 décembre 1938.

84 Ibid., rapport de l'IA de Metz sur l'académie de Strasbourg, 3 janvier 1939.

85 Cf. la première phrase de cet article.

86 Ibid., rapport de l'IA d'Evreux pour le conseil académique de Caen, année 1937-1938. 
L'inspecteur de l'Ardèche, pour l'académie de Grenoble, signale aussi "la nécessité de faire appel parfois à des spécialistes, artistes, artisans car les professeurs ne sont pas omniscients. D’où la nécessité de disposer de ressources supérieures à celles que peuvent fournir les heures supplémentaires ou les coopératives des élèves et les dons ${ }^{87}$ ". L'inspecteur de la Meurtheet-Moselle, pour l'académie de Nancy, insiste sur " la bonne volonté des enseignants dans cette année inaugurale ${ }^{88}$ " mais reconnaît que ce zèle est en train de se ralentir. Beaucoup de proviseurs et d'inspecteurs notent également que l'on ne s'improvise pas animateur de groupes, même si l'on est un enseignant chevronné. L'inspecteur d'académie de Lyon écrit, le 8 décembre 1938, que " rares sont les professeurs qui ont des talents extra-scolaires personnels leur permettant de capter et de retenir en dehors de la classe l'attention des élèves [...]. Beaucoup donnent un enseignement d'un caractère abstrait et n'ont pas les aptitudes à diriger des excursions géologiques, botaniques, artistiques, des visites d'usines ou d'exploitations. Beaucoup ont aussi l'habitude du travail solitaire et [...] éprouvent quelques craintes à l'idée de conduire au dehors des élèves qui sont agités ${ }^{89}$ ".

L'inspecteur de la Roche-sur-Yon, le 23 novembre 1938, dans son rapport sur l'académie de Poitiers, pointe de nombreuses difficultés :

"La réalisation n'a pas toujours été facile. Il a fallu vaincre l'hostilité des familles auxquelles les occupations facultatives semblent inutiles, retenir l'attention des élèves par une variété incessante des occupations, se débattre au milieu des difficultés financières créées par la nécessité d'un matériel même rudimentaire, enfin rechercher parmi le personnel des maitres de bonne volonté capables de s'intéresser à l'innovation et d'en comprendre le but " ${ }^{90}$.

Dès lors, faute de volontaires motivés, les chefs d'établissement sont obligés d'avoir recours aux collègues indifférents qui sont en sous-service ou aux surveillants. Dans l'académie de Strasbourg, le rapport insiste sur la participation contrainte de " certains enseignants dont le maximum d'heures n'est pas atteint 91 ". L'inspecteur de l'Ain, dans son rapport sur l'académie de Lyon de décembre 1939, note :

87 Ibid., rapport de l'IA de l'Ardèche sur l'académie de Grenoble en 1937-1938, 1er décembre 1938.

88 Ibid., rapport de l'IA de la Meurthe-et-Moselle sur l'académie de Nancy, 5 décembre 1938.

89 Ibid., rapport de l'IA de Lyon sur l'académie de Lyon, 8 décembre 1938.

90 Ibid., rapport de l'IA de La Roche-sur-Yon sur l'académie de Poitiers, 23 novembre 1938.

91 Ibid., rapport de l'IA de Metz sur l'académie de Strasbourg, 3 janvier 1939. 
"l'excellence du principe ne doit pas nous empêcher de dire, en toute vérité, que la formule actuelle des loisirs dirigés, n'excite guère l'enthousiasme du personnel. Je ne retrouve pas, dans les rapports des chefs d'établissement, l'écho même affaibli de certains articles de la Revue universitaire " ${ }^{92}$.

Il insiste alors sur les programmes bien trop lourds pour permettre la neutralisation du samedi après-midi, sur la nécessité aussi du travail personnel des élèves. Du côté enseignant, beaucoup d'hommes sont engagés le samedi après-midi dans d'autres activités, alors que les femmes enseignantes, souvent mariées, ont besoin de cette demi-journée pour la consacrer à leur famille.

Le conseil académique de Lille, en décembre 1938, apparaît réservé sur " cette initiative originale qui représente un essai encore un peu timide pour acclimater dans l'enseignement secondaire les méthodes actives de la pédagogie nouvelle ${ }^{93}$ ". On compte sur l'intérêt que présente le libre choix d'une activité pour susciter l'effort de l'élève. " En fait, le premier enthousiasme passé, il y a eu beaucoup de déchet. Il en est généralement ainsi pour toute nouveauté mais plus particulièrement pour celles qui se fondent sur la liberté ". Le recteur Georges Hardy note deux effets positifs, cependant. Le choix des activités étant libre, les élèves ont été mélangés, quels que soient leurs âges, classes et niveaux. Il insiste également sur " les possibilités insoupçonnées qu'on trouve chez les élèves quand on s'adresse à leur intérêt profond et à leur libre initiative ». Il faut aussi, signalent plusieurs enseignants, relativiser la nouveauté de ces loisirs dirigés. Certes l'expérimentation systématisée au sein des collèges et des lycées est neuve mais la pratique, elle, est ancienne. De nombreux enseignants n'ont pas attendu les loisirs dirigés pour utiliser les méthodes actives. C'est ce que relève Mademoiselle Divin, professeur au lycée Lamartine, dans la Revue universitaire. " La circulaire ministérielle sur les loisirs organisés du samedi a été généralement bien accueillie ${ }^{94}$ ", mais, note-t-elle, il n'y a là rien de nouveau.

"Il s'agit simplement d'imposer partout des méthodes employées depuis longtemps par des gens qu'on accusait de dévouement parce que les dites méthodes exigent beaucoup de temps, de travail et de bonne humeur. Tout ce qui est proposé par le ministre de l'Éducation nationale en matière de distractions apportées aux élèves du samedi - lectures, promenades, visites d'usines et de monuments, représentations - s'est déjà fait, surtout je crois, chez les jeunes filles. [... Il est] piquant de voir officiellement reconnue l'utilité de pratiques qu'on jugeait inspirées par la frivolité et l'envie de ne rien faire ".

92 Ibid., rapport de l'IA de l'Ain sur l'académie de Lyon, 18 décembre 1939.

93 Archives départementales du Nord, 2 T 139 , conseil académique de Lille, séances et rapports de décembre 1938.

94 M. Divin, "À propos des loisirs dirigés ", Revue universitaire, t. 2, 1937, p. 416-418. 
Même si cet aspect n'apparaît pas dans les rapports des inspecteurs et des proviseurs, il est évident que cette initiative réformatrice rencontre une hostilité certaine de la part des enseignants les plus conservateurs. Face à la montée progressive des effectifs des collèges et des lycées à partir de l'instauration de la gratuité, entre 1928 et 1933, certains enseignants dénoncent la baisse du niveau et les dangers qui menacent les humanités classiques, fondements de la culture secondaire. Hostiles aux revendications des partisans de l'École unique, voulant conserver des filières sélectives même s'ils reconnaissent les bienfaits de la méritocratie fondée sur la sévère sélection d'une élite de l'intelligence et non de la fortune, ils adoptent une position corporative de défense des intérêts d'un enseignement secondaire qu'ils estiment menacé de primarisation. Dès lors les " loisirs dirigés " et leur volonté de promouvoir les méthodes actives leur apparaissent comme une arme habilement maniée par "le camp des primaires ", en particulier le Syndicat national des instituteurs, pour affaiblir le secondaire. Il en va alors du respect de leur distinction et des spécificités de l'ordre secondaire. La puissante Société des agrégés, fondée en 1914 et qui regroupe en 193987 \% des professeurs agrégés en poste dans l'enseignement secondaire ${ }^{95}$, est ainsi rapidement hostile à la réforme de Jean Zay qui, selon eux, mutile la culture classique. Cette innovation pédagogique, analysée comme symbolique des intentions cachées du ministère, ravive les clivages corporatifs et catégoriels.

\section{3 - Innovation pédagogique et enjeux politiques}

Les loisirs dirigés vont également être victimes du rejet plus global et souvent radical de la politique du Front populaire. L'innovation pédagogique sert ici de prétexte aux querelles politiques et se retrouve transformée en argument politicien, les loisirs dirigés étant lus comme un révélateur des projets néfastes du gouvernement. À droite et à l'extrême droite, ils sont systématiquement dénoncés comme l'un des signes de la déraison du Front populaire. Gringoire consacre peu de temps aux réformes scolaires de Jean Zay, " ministre de la démence nationale ${ }^{96}$ ", et préfère dénoncer " Blum la guerre ${ }^{97}$ " et sa participation au complot marxiste qui menace la France. Robert Brasillach condamne les quinze à vingt mois qui suivent mai 1936 comme

95 Yves Verneuil, Les agrégés. Histoire d'une exception française, Paris, Belin, 2005, p. 144 (les agrégés représentent 93,6\% du personnel enseignant des 18 lycées de Seine et de Seine et Oise à la fin des années 1930 pour 46,8 \% des personnels des 115 lycées des départements). 
l'une des périodes " les plus nuisibles ${ }^{98}$ " que la France ait connue. Il y perçoit la " primauté de la bassesse " qui, " par une sorte d'étrange sadisme ", donne le ministère de l'Éducation nationale " à un antimilitariste notable, devenu belliciste depuis ${ }^{99}$ ". Pendant l'Occupation, René Benjamin, qui soutient l'œuvre du Maréchal Pétain, dénonce dans Vérités et rêveries sur l'éducation ${ }^{100}$ l'école de la Troisième République qui, selon lui, s'est fourvoyée dans l'entre-deuxguerres. On a oublié les fondamentaux de la culture classique, les auteurs latins et grecs, et l'on a renoncé à l'effort. " Une classe restera caractéristique de cette époque de comédie qui devait finir tragiquement, la classe des loisirs dirigés ${ }^{101}$ ". Dans ces temps "bolchevistes ", la gratuité fut " un champignon, une moisissure ", qui a tout nivelé. L'École unique promue par Jean Zay, " assisté des loges et de la CGT ${ }^{102}$ ", a tout fait pour détruire l'enseignement traditionnel, réservé à une élite et constructeur de l'identité française.

Moins radicaux, d'autres journaux attaquent pourtant les réformes scolaires du Front populaire. Jean Guiraud, dans La Croix, dénonce les maîtres laïques qui deviennent " des amuseurs ${ }^{103}$ ". De nombreux hommes politiques de droite dénoncent cette œuvre anti-nationale qui assure la promotion des loisirs contre les études sérieuses. On demande le déplacement des loisirs dirigés au jeudi après-midi, quand les classes sont vacantes, afin de ne pas perdre le samedi après-midi. La Revue de Paris, dans son numéro du 15 juin 1938, décrit "Les expériences de l’Éducation nationale ${ }^{104}$ ", dénonçant le ministre Jean Zay qui, " après s'être accordé les pleins pouvoirs, applique par décrets son projet de loi sur la réforme de l'enseignement " et pratique la politique du " fait accompli " par la ruse de l'expérimentation. "Le ministre de l'Éducation nationale a donné des vacances à la légalité. Il légifère lui-même ". Le projet de loi de mars 1937 n'ayant pas été examiné, le fait que Jean Zay procède par décrets et arrêtés est jugé illégal, la réforme devant passer par le vote d'une loi débattue et acceptée par les deux assemblées. Dès lors les loisirs dirigés sont désignés

98 Robert Brasillach, Une génération dans l'orage. Notre avant-guerre, Mémoires, Le Livre de Poche, 1992, p. 233.

99 Ibid. p. 245.

100 René Benjamin, Vérités et rêveries sur l'éducation, Paris, Plon, 1941, 242 p.

101 Ibid., p. 136.

102 Ibid., p. 152.

103 La Croix du 28 janvier 1938.

104 Léon Blum, "Les expériences de l'Éducation nationale ", La Revue de Paris du 15 juin 1938, p. 913 et suivantes. 
comme symboliques de la volonté de dictature marxiste que veut imposer le Front populaire.

L'objectif de ces innovations pédagogiques morcelées, selon l'auteur de l'article, (Léon Blum, homonyme du président du Conseil, professeur honoraire au lycée Janson de Sailly) est de combattre l'enseignement classique. Le ministre applique des réformes dangereuses et inacceptables " dictées par la CGT [...] qui veut nationaliser la fabrication des esprits comme les usines ". Il y a, selon l'auteur, un plan politique derrière tout cela, visant à la prise du pouvoir par les " primaires " qui veulent détruire l'ordre secondaire. "C'est l'arme forgée par les syndicats primaires pour la lutte des classes par le moyen de l'école ${ }^{105}$ ". La vague de paresse qui déferle sur les travailleurs va-t-elle submerger la jeunesse qui s'instruit ? "L'enseignement secondaire dépérit par l'excès des loisirs ". Le même chroniqueur revient à la charge dans la Revue de Paris

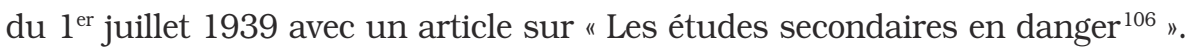
Il dénonce de nouveau la tactique de Jean Zay, qui passe outre le vote d'une loi et multiplie les décrets et les arrêtés. Alors que les menaces grondent à nos frontières, "l'Éducation nationale s'acharne chez nous à la ruine des études qui ont contribué pour une large part à faire de la France la patrie des droits de l'Homme, l'espoir des peuples qui veulent rester libres [...]. Il s'agit de satisfaire les syndicats cégétistes préoccupés d'unifier, c'est-à-dire de niveler d'abord les divers enseignements puis les catégories de maitres et leurs traitements ".

" Jean Zay soutient la gageure de paralyser le travail encore davantage. Quatre mois de vacances ou de congés, deux jours par semaine le reste de l'année, n'offraient pas de loisirs suffisants à la jeunesse des lycées et des collèges. Il y ajoute une demi-journée le samedi [...]. Il en résulte qu'il faut entasser dans le reste de la semaine des heures d'enseignement déjà excessives. [...]. Si la direction des loisirs paraît à certains une pédagogie recommandable, si l'organisation de plaisirs intelligemment conduits peut, en effet, contribuer à l'éducation, si l'on juge que l'Université doit s'en charger, et si l'on accepte de grever encore lourdement son budget, pourquoi amputer pour lui faire place, le temps consacré à la classe quand le jeudi est tout indiqué pour cet essai [...]. Ainsi à l'ancienne Instruction publique, le ministre inaugure le département de la récréation nationale, en même temps qu'il applique le plan d'études cégétiste. Il affaiblit toutes les disciplines en même temps qu'il poursuit la ruine de l'enseignement secondaire " 107 .

105 Ibid., p. 922.

106 Léon Blum, "Les études secondaires en danger : l'application d'un projet de loi mort né ", La Revue de Paris du $1^{\text {er }}$ juillet 1939, p. 149-170.

107 Ibid., p. 168-169. 
Dans Le Temps du 28 mars 1937, le journaliste parle d'une " opération de nivellement général de la France ${ }^{108}$ ". Dans le numéro du $1^{\text {er }}$ janvier 1938, il décrit deux expériences de loisirs dirigés et conclut en fustigeant une Éducation nationale qui ne désirerait plus que former des "cabotines, des romanciers et des reporteurs ${ }^{109}$ ". Jean Zay lui-même reconnaît d'ailleurs l'erreur faite dans le choix du terme et l'amalgame qui est immédiatement fait avec la politique des congés payés et de promotion des loisirs ouvriers.

" Notre malheur fut peut-être d'avoir employé d'abord sans méfiance le terme "loisirs dirigés". Loisirs ! Cela vous avait un petit parfum de paresse, de nonchalance, de goût du moindre effort. En 1936, la situation sociale, grèves et revendications ouvrières, réduction de la journée de travail, rejaillissaient singulièrement sur l'opinion qu'on se faisait des réformes scolaires. Il était entendu qu'on ne voulait plus travailler en France [...]. Nous précisâmes notre pensée en disant désormais " activités dirigées " [...]. Réformateurs, méfiez-vous de vos étiquettes ${ }^{110}$.

Sur la gauche de l'échiquier politique, l'unanimité n'existe pas. Certes, Célestin Freinet salue d'abord les loisirs dirigés et leur déclinaison dans l'enseignement primaire que sont les " activités dirigées ". Après avoir critiqué la surcharge des horaires, il présente les loisirs dirigés comme " un frein officiel à la folie de la surcharge des programmes et des examens [....]. Nous ne voudrions pas avoir l'air d'encenser un ministre Front populaire mais nous devons affirmer à présent notre satisfaction presque totale à la lecture de la circulaire ${ }^{111}$ ". Freinet salue surtout la démarche expérimentale choisie, qui laisse une large part d'initiative au terrain local. Dès lors, l'expérience est " comme un coin d'éducation nouvelle enfoncé en plein cœur de l'école ${ }^{112}$ ". Mais, très vite, le pédagogue comprend que la réforme est asphyxiée par l'opposition larvée ou directe des enseignants, et aussi par la faiblesse des crédits. L'Éducateur prolétarien du 15 juin 1939 est ainsi beaucoup plus mesuré. Le soutien est toujours là, mais Freinet demande un maximum d'efficacité.

"Pour aboutir, il faut en effet que les activités dirigées ne soient pas qu'un pur verbiage [...]. Les activités dirigées ne doivent pas être de paisibles et anodines heures de bricolage et de travail actif, jetées en travers de l'horaire traditionnel comme les dix minutes de récréation au milieu de trop longues heures de classe [...].

108 Archives nationales, 312 AP 5, Papiers Abraham, Le Temps du 28 mars 1937.

109 Le Temps du $1^{\text {er }}$ janvier 1938.

110 Jean Zay, Souvenirs et solitude, op. cit., p. 175.

111 Célestin Freinet, L'Éducateur prolétarien, 13e année, 30 novembre 1937, p. 73-79.

112 Ibid., p. 78. 
Nous n’avons cessé de dénoncer cette tendance scolastique qui consiste à appliquer passivement les instructions ministérielles et à remplir les heures prévues par des activités quelconques sans lien d'aucune sorte avec la vie et les intérêts des enfants $" 113$.

Les activités dirigées doivent être le levain de la rénovation de l'enseignement et Freinet réclame des réalisations coordonnées qui permettent de faire avancer la cause de l'école démocratique. L'extrême gauche politique elle-même dénonce un effet d'annonce car la réforme n'est pas suivie d'un plan financier d'accompagnement. L'Université syndicaliste du $1^{\mathrm{er}}$ décembre 1937 manifeste son scepticisme et Georges Cogniot ${ }^{114}$, proche collaborateur de Maurice Thorez au sein du Parti communiste français, rédacteur en chef de L'Humanité et spécialiste des questions d'éducation, s'affirme plus préoccupé par les mesures de démocratisation de l'enseignement, d'augmentation du nombre des postes et de revalorisation des carrières que par la rénovation pédagogique, insistant sur les limites financières de la réforme alors que la Guerre d'Espagne mobilise aussi les esprits.

Pendant deux années scolaires, d'octobre 1937 à juillet 1939, les établissements secondaires français multiplient les initiatives pour mettre en œuvre l'arrêté du 22 mai 1937 fondant les loisirs dirigés. En puisant dans les pratiques du scoutisme mais aussi des mouvements de l'Éducation nouvelle, il s'agit d'acclimater dans l'Éducation nationale leurs méthodes actives en fondant un espace de libre initiative fixé au samedi après-midi. Dans sa circulaire du 26 avril 1939, Jean Zay relève ainsi que " nous comprenons, sous le terme d'activités dirigées, une méthode d'éducation fondée sur les progrès de la psychologie de l'enfant. Elle respecte et utilise, en la guidant, sa spontanéité ${ }^{115}$ ". L'idée est de faciliter, à partir de ce temps

113 Célestin Freinet, "Activités dirigées ", L’Éducateur prolétarien, n 18, 15 juin 1939, p. 401-404.

114 Georges Cogniot (1901-1978) est élève de l'École normale supérieure (promotion 1921) et agrégé de lettres (1924). Enseignant dans le secondaire, il devient un militant communiste très actif qui participe à la création de l'Internationale des travailleurs de l'Enseignement. Élu député de Paris en 1936, il est le rapporteur du budget de l'Éducation nationale à la Chambre en 1937. Il ne cesse de protester contre l'insuffisance des crédits accordés à l'École, exigeant la mise en place d'une réelle école unique et démocratique. Dans ses mémoires, il écrit à propos du projet scolaire de Jean Zay : "la principale critique que le projet suscita d'emblée portait cependant sur un autre point [...] : son architecture assez impressionnante ne reposait absolument sur aucune assise financière. Il était difficile de prendre tout à fait au sérieux des propositions que n'accompagnait aucune référence aux moyens nécessaires à leur application " (Georges Cogniot, Parti pris, t. I, D’une guerre à l'autre. Cinquante ans au service de l'humanisme réel, Paris, Les Éditions sociales, 1976, p. 302).

115 Archives nationales, 312 AP 6, Papiers Abraham, circulaire du 26 avril 1939 aux recteurs. 
scolaire spécifique, la diffusion de germes de novation susceptibles d'engager la rénovation de l'ensemble du système éducatif. Les clubs, les associations, les troupes théâtrales, les activités artistiques, les sorties et visites de musées, de fermes ou d'usines se multiplient tout comme les activités liées au cinématographe ou à la TSF. "L'appel d'air ", pour reprendre les propos de Jean Zay, est bien au rendez-vous mais les pesanteurs du système réduisent l'impact de la réforme.

À travers cette introduction des loisirs dirigés, ce sont les dynamiques de l'innovation pédagogique et de la réforme éducative qui sont à l'œuvre et qui sont interrogées dans toute leur complexité. Cinq remarques générales se dégagent de cette étude de cas. Il apparaît tout d'abord qu'innovation pédagogique et réforme éducative, si elles peuvent parfois marcher de concert, fonctionnent souvent de manière décalée au sein du système éducatif. Les grandes réformes éducatives qui passent par la loi entraînent de profonds bouleversements structurels qui monopolisent à la fois les préoccupations des acteurs et les moyens humains et financiers, les enjeux pédagogiques étant placés pour un temps à l'arrière-plan. Un " environnement dérangeant ${ }^{116}$ " est souvent nécessaire pour susciter des engagements novateurs et mobiliser des stratégies nouvelles. C'est parce que le très ambitieux plan de réforme de l'enseignement de Jean Zay, présenté en mars 1937, est en panne que son cabinet procède à des expérimentations pour affirmer sa volonté réformatrice maintenue et tenter le changement pédagogique sans la réforme.

Il faut ensuite relever le poids des logiques scolaires et la force du cadre de la classe. Si les loisirs dirigés sont mis en place pour permettre de travailler autrement et de briser le cadre scolaire classique, ce dernier ne cesse de les attirer vers lui pour leur faire perdre leur originalité première dans une sorte de contamination scolaire de l'innovation. C'est ainsi que les loisirs dirigés qui se maintiennent dans le temps sont souvent les plus proches des activités disciplinaires, alors que les logiques propres à l'institution, en particulier celles de la gestion des services des personnels enseignants et de l'organisation des activités au sein de l'établissement, imposent leurs contraintes. Les clivages catégoriels au sein des personnels, en particulier enseignants, sont aussi des éléments importants de l'acceptation ou du refus de l'innovation éduca-

116 Marcel Grandière, "Introduction. L'innovation et l'éducation " dans Marcel Grandière et Agnès Lahalle (dir.), L'innovation dans l'enseignement français (XVI'-XX'e siècles), Nantes, SCEREN-CRDP Pays de la Loire/INRP, 2004, p. 18. 
tive. L'innovation, pour être acceptée doit répondre à des problèmes ressentis par les enseignants dans leur travail au quotidien. Les familles elles-mêmes participent à ce recadrage scolaire de l'innovation, refusant ce qu'elles estiment être une perte de temps dans l'investissement éducatif de leurs enfants. Les élèves quant à eux, jouent très vite de la dimension optionnelle des loisirs dirigés pour s'en abstenir, arguant de la nécessité de disposer de temps pour leur travail scolaire hebdomadaire. Comme le note Marcel Grandière, "il faut aussi à l'innovation un espace de mise en exercice. L'histoire éducative montre que cet espace n'est pas facile à trouver ${ }^{117}$ ". Les loisirs dirigés n'échappent pas à cette règle, les pressions se faisant très fortes pour récupérer le samedi aprèsmidi pour le travail scolaire, au nom de la réussite des élèves.

Les loisirs dirigés et leur brève histoire démontrent, c'est le troisième point, que l'innovation pédagogique ne vit pas que d'amour et d'idées fraîches. Toute innovation a un coût et les loisirs dirigés sont très vite mis à mal par le manque de moyens autant que par les oppositions pédagogiques ou politiques qu'ils rencontrent. Philippe Savoie a bien montré combien les clivages statutaires et les contraintes financières pèsent sur l'évolution du métier d'enseignant ${ }^{118}$. En ce qui concerne les loisirs dirigés, les établissements comprennent vite qu'ils ne disposent pas des moyens financiers nécessaires et les chefs d'établissement doivent souvent utiliser les heures de loisirs dirigés pour compléter les services de collègues peu intéressés alors que les enseignants volontaires se lassent du bénévolat. Les bricolages locaux qui se mettent en place ne suffisent pas et épuisent rapidement les hommes de bonne volonté. Il faut également relever, quatrième point, que le temps " court " du politique et de ses querelles partisanes interfère très souvent sur le processus d'innovation pédagogique et de réforme éducative. L'innovation a besoin de temps pour se développer, pour convaincre à la fois les familles, les élèves et les enseignants de ses bienfaits, mais elle dispose rarement de ce temps long nécessaire à son épanouissement. Les querelles partisanes se saisissent avec délectation des questions éducatives qui ont le grand mérite de parler à tout le monde et d'engager à la fois le passé de la nation et son avenir par la formation de sa jeunesse. Célébrée par les for-

117 Ibid., p. 20.

118 Philippe Savoie, "Les enseignants du secondaire (XIX -début XX siècle) : L'évolution du métier ", dans Marcel Grandière et Agnès Lahalle (dir.), L'innovation dans l'enseignement français..., op. cit., p. 129-144. 
ces au pouvoir, responsables de son lancement, l'innovation pédagogique est décriée par les opposants et instrumentalisée à des fins politiques. Le cas des loisirs dirigés, lancés dans un contexte d'affrontement politique particulièrement vif, en fournit un exemple frappant. Si, pour le camp conservateur, les loisirs dirigés révèlent au grand jour le fait que l'Éducation nationale est devenue le ministère de la " récréation nationale ", dirigée par des complices de Moscou, ils apparaissent également insuffisants à l'extrême gauche pédagogique qui découvre la faiblesse des moyens dégagés pour leur mise en œuvre et le fait qu'ils sont l'arbre aux jolis fruits qui cache la forêt des problèmes non résolus et de l'injustice scolaire.

La dernière remarque concerne la prise de décision en éducation. L'innovation pédagogique peut-elle se décréter et s'imposer aux acteurs éducatifs par la volonté expresse du pouvoir central ? Comment piloter le développement de pratiques pédagogiques innovantes alors que celles-ci nécessitent autonomie et adaptation aux contextes locaux et aux ressources humaines mobilisables sur le terrain, et exigent le tâtonnement expérimental et l'ajustement permanent? Le cas des loisirs dirigés montre combien l'équilibre est délicat à trouver entre la tentation d'un cadrage strict, lié à une impulsion énergique venue du ministère qui peut vite se transformer en dirigisme, et la possibilité d'un guidage ministériel plus distant, qui fixe les finalités et le cadre général de l'innovation pédagogique, mais laisse ensuite une grande latitude au terrain pour organiser et gérer ces innovations, au risque d'être perçu comme une absence de soutien réel, surtout quand il s'accompagne d'une aide financière dérisoire. Le principe d'activités personnalisées laissant une large place à l'initiative de l'élève est apparu à nouveau à de nombreuses reprises dans l'enseignement secondaire, qu'il s'agisse des activités dirigées lancées dans les classes nouvelles de sixième en 1945 avec davantage de moyens ${ }^{119}$, ou plus près de nous, des travaux personnels encadrés

119 Jean-Michel Chapoulie, L'école d'État conquiert la France. Deux siècles de politique scolaire, Rennes, Presses universitaires de Rennes, 2010, p. 366-369. Ces classes nouvelles sont 185 implantées dans 139 établissements en 1945 et 844 implantées dans 199 établissements en décembre 1949. Le départ à la retraite de Gustave Monod en 1951 signifie leur suppression. Voir aussi Antoine Savoye, "Réforme pédagogique, réforme disciplinaire : l'expérience des classes nouvelles dans l'enseignement du second degré (1945-1952) ", dans Renaud d’Enfert et Pierre Kahn (dir.), En attendant la réforme. Disciplines scolaires et politiques éducatives sous la IVe République, Grenoble, Presses universitaires de Grenoble, 2010, p. 51-64. 
en lycée ${ }^{120}$, à partir de 2000, ou des itinéraires de découvertes en collège ${ }^{121}$, à partir de 2002. Ces expériences ont, dans l'ensemble, levé les mêmes espérances et rencontré les mêmes difficultés que les loisirs dirigés de la fin des années 1930.

Jean-François CONDETTE

Université d'Artois, Laboratoire CREHS (EA 4027) jeanfrancois.condette@wanadoo.fr

120 Les travaux personnels encadrés (TPE) sont introduits à titre expérimental en classe de première en 1999-2000 puis généralisés en 2000-2001 en première. Ils sont facultatifs en terminale (2001-2002) puis généralisés en 2002-2003 mais supprimés dans cette classe en 2005-2006. La note de service du 20 octobre 2005 relève que " les TPE offrent aux élèves l'occasion de développer des capacités d'autonomie et d'initiative dans la conduite de leur travail ".

121 C'est l'arrêté du 14 janvier 2002 qui crée les Itinéraires de découvertes (IDD) en classes de cinquième et de quatrième, à raison de deux heures par semaine. Comme l'indique la circulaire du 2 août 2002, ils sont introduits afin de " permettre aux élèves de s'investir dans des projets interdisciplinaires et de travailler de façon autonome, individuellement ou en groupe ". 